\title{
Design and realization of the evaluation of expert reputation in the online evaluation system for the master dissertation
}

\author{
Huang Lin $^{1,}$, Wang Shuhai ${ }^{2, \mathrm{~b}}$, Fan $\mathrm{Yu}^{1}$ \\ ${ }^{1}$ School of Graduate Shijiazhuang Tiedao University, Shijiazhuang, China \\ ${ }^{2}$ School of Continuing Education Shijiazhuang Tiedao University ,Shijiazhuang, China \\ ${ }^{a}$ E-mail:yjspyb@stdu.edu.cn , b E-mail:wangsh@stdu.edu.cn
}

Keywords: Master dissertation, Online evaluation, Reputation evaluation

\begin{abstract}
: issertation comprehensively reflects the research capacity, innovation ability and literary ability of a postgraduate, and the dissertation evaluation is to give an objective and fair evaluation of the master dissertation. Hence the expert selection and the evaluation of its work are extremely important parts of the dissertation evaluation, and they affect the objectivity, fairness and the academic level of the results. In this paper, evaluation system for expert reputation based on the points system is designed. At the initial stage, an expert is evaluated by his basic information. Then after several rounds of review, the new evaluation is a comprehensive result of every review, and gradually approaches the actual situation of the expert. The system is proved to be feasible via actual case studies.
\end{abstract}

\section{Introduction}

To ensure the quality of the master dissertation, the graduate cultivation departments have established their own systems. The developed countries have basically realized online evaluation for the master dissertation at present. In the universities of shanghai, all the master dissertations have been evaluated online anonymously now.

To find an appropriate expert is a distinctly important part of the dissertation evaluation. However, because of the tedious procedures and work, some universities are not willing to evaluate dissertation from other colleges. Besides, some experts only choose to evaluate dissertation from universities entrusted, and most of their work was arranged by the postgraduate teaching secretary, so it's very difficult to find a suitable expert. On the other hand, because of lacking of restraint mechanism, a lot of experts do not pay enough attention to the evaluation and thus it's hard to guarantee the quality of the dissertation evaluation.

Considering of all these questions, an evaluation system of the expert reputation is designed to solve them efficiently. Based on the academic level, evaluation experience and the organizers' evaluation for an expert, the evaluation of the expert reputation system is established to provide a basis for how to select expert for the dissertation evaluation by taking advantage of the points system.

\section{Design of the evaluation of the expert reputation system}

In the recent years, evaluation methods such as the shopping online and QQ user rankings are widely accepted. All these methods make use of the points system, and it turns out to be convenient and vivid. By taking full advantage of these successful experiences, the evaluation of the expert reputation system is established based on the points system.

\section{Design principles:}

a. Include as much attribute information and behavior information relevant to the expert reputation as possible.

b. Easy to operate and the result is practical.

c. Evaluation result can not only shows the expert reputation in some point, but also reflects the comprehensive expert reputation. 
d. The reputation level is cumulative and should be updated dynamically.

e. Highlight the key point, give priority to the qualitative analysis, and combine the quantitative analysis as an auxiliary.

\section{Evaluation index system}

There are many attribute and behavior information that would affect the level of the expert reputation, but it mainly reflects in three aspects:

a. The academic level of the expert

The academic level of the expert is a very important index to reflect the expert's ability, and it should take several factors into consideration, such as the expert's title, degree of education, highest degree, whether doctoral tutor/academician or not. Some other information like the academic achievement can also reflect the academic level of the expert. However, considering that the information is difficult to quantify and will bring out many problems in period of calculation. therefore it is not taken into consideration.

b. The review experience of the expert

The review experience of expert is mainly reflected by the times that the expert participates in the dissertation evaluation and the number of dissertations that have been evaluated every time. In this paper, the more the expert joins in and the larger the number of dissertations that have been evaluated, the richer the review experience of the expert is, which means that the experience points is higher.

c. The organizers' evaluation to the expert

The organizers are users of the dissertation evaluation, so the evaluation works only when users are satisfied. However, it's hard to make the users would like to evaluate the expert, to realize it in reality is difficult as well. To guarantee that organizers could evaluate the expert in accordance with the standard, the system should be able to record information about the review work and evaluate it automatically, and it is used as a reference to evaluate the organizers.

According to the analysis above, the evaluation index system of the expert reputation is established in table 1:

Table 1 the evaluation index system of the expert reputation

\begin{tabular}{cll}
\hline First-class target & Second-class target & \multicolumn{1}{c}{ Standard for evaluation } \\
\hline \multirow{3}{*}{$\begin{array}{c}\text { 1.academic level } \\
\text { (Z1) }\end{array}$} & 1.1 title(Z11) & Quantify according to senior, sub-senior, etc. \\
\cline { 2 - 3 } & $\begin{array}{c}\text { 1.2 degree(Z12) } \\
\text { 1.3 degree of education (Z13) }\end{array}$ & Quantify according to doctor, master, etc. \\
\cline { 2 - 3 } & Quantify according to doctor-postgraduate, ostgraduate, etc. \\
\cline { 2 - 3 } 2.1.5 doctoral tutor(Z15) & Quantify according to whether academician or not \\
\hline (Z2) & $\begin{array}{l}\text { Q.1 number of evaluated } \\
\text { dissertations (Z21) }\end{array}$ & $\begin{array}{l}\text { Quantify according to reviewing master dissertation or doctor } \\
\text { dissertation }\end{array}$ \\
\hline \multirow{2}{*}{ 3.evaluation (Z3) } & $\begin{array}{l}\text { 3.1 evaluation from organizers } \\
\text { (Z31) }\end{array}$ & $\begin{array}{l}\text { Quantify according to the number getting praise, ordinary, or bad } \\
\text { review. }\end{array}$ \\
\hline
\end{tabular}

\section{Design of the quantification method}

In accordance with the indexes above, base score, experience score, evaluation score and comprehensive score are set for every expert. Base score is given according to the academic level of the expert, experience score is given according to the review experience, evaluation score is given according to the evaluation from organizers. Synthesis score is a combination of base score, experience score and evaluation score. The marking scheme is shown as following:

a. Base score (S1)

The base score is set according to the initial score (S0) and the academic level of the expert. Score of academic level is a summation of quantitative score of title (S11), degree (S12), degree of education (S13), doctoral tutor (S14) and academician (S15). That is: $S_{1}=S_{0}+\sum_{i=1}^{5} s_{1 i}$

Title quantification score (S11): quantify attribute information about title of the expert, record senior as S111, sub-senior as S112, others as S113.

Degree quantification score (S12): quantify attribute information about highest degree of the expert, record doctor as S121, master as S122, others as S123.

Degree of education quantification score (S13): quantify attribute information about degree of 
education, record doctor-postgraduate as S131, postgraduate as S132, and others as S133.

Academician quantification score (S14): quantify attribute information about that whether the expert is academician or not, record academician as S141, and others as S142.

Doctoral tutor quantification score (S15): quantify attribute information about that whether the expert is doctoral tutor or not, record doctoral tutor as S151, and others as S152.

b. Experience score (S2):

The experience score is set according to the evaluation experience of the expert. It is set according to the number of dissertation evaluated (S21). Record the score of evaluating a piece of master dissertation as $\mathrm{S} 21_{1}$, and a doctor's one as $\mathrm{S} 21_{2}$. That is:

$S 2=S 21=S 21_{1} \times C 1+S 21_{2} \times C 2$

Where C1 stands for the number of evaluated master dissertations; C2 stands for the number of evaluated doctor dissertations.

c. Evaluation score (S3):

The evaluation score is set according to the score given by the organizers. Expert would get an assessment after the dissertation evaluation every time, and record praise as $\mathrm{S} 31_{1}$, ordinary as $\mathrm{S} 31_{2}$, bad review as S31 3 . That is: $S 3=S 31=S 31_{1} \times P 1+S 31_{2} \times P 2+S 31_{3} \times P 3$

Where P1 stands for the number of evaluations that receive praise; P2 stands for the number of evaluations that receive an ordinary assessment; P3 stands for the number of evaluations that receive bad review.

d. $\quad$ Synthesis score (S):

The synthesis score is summation of base score, experience score, evaluation score of the expert. That is: $\mathrm{S}=\mathrm{S} 1+\mathrm{S} 2+\mathrm{S} 3$

\section{Design of the evaluating parameter table}

a. evaluating parameter table

This table is used to save second-class score that related to the first-class score about the academic level, that is $\mathrm{S}_{\mathrm{i}} \mathrm{i}_{\mathrm{k}}, \mathrm{i}=1 \ldots \ldots . . \mathrm{k}$ is set according to the number of Indexes quantified. The initial score is set by management, and would be updated all the time when system runs, besides, initial score (S0) set by system should be saved separately. The metadata description of the evaluating parameter table is shown in table2.

Table 2 T_ZJ_PJCS : Evaluating parameter table

\begin{tabular}{|c|c|c|c|c|c|}
\hline property & Name & $\begin{array}{c}\text { Data type and } \\
\text { length }\end{array}$ & Null or not & $\begin{array}{c}\text { Primary/exter } \\
\text { nal key }\end{array}$ & remark \\
\hline $\bar{F}$ F_Id & Serial number & Integer & $\mathrm{N}$ & $\mathrm{P}$ & \\
\hline F_PJZBBH & Number of evaluation index & Char(10) & $\mathrm{N}$ & & Value : Z11、12... \\
\hline F_PJZBMS & description of evaluation index & Varchar(20) & $\mathrm{N}$ & & For example: degree" \\
\hline F_LHXH & Serial number quantification & Integer & $\mathrm{N}$ & & Value: $1 、 2 \ldots$ \\
\hline F_LHMS & Description quantification & Varchar(100) & & & For example: doctor, master... \\
\hline F_LHFZ & score quantification & Integer & & & For example: 10、 5 \\
\hline
\end{tabular}

b. $\quad$ Parameter quantification mapping table

Relationship should be established between the expert's academic level like title, degree, education degree and the quantization level of the expert in the evaluation index system of the expert reputation. Take title, as an example: professor $\rightarrow$ is equivalent to senior, researcher $\rightarrow$ is equivalent to senior, senior engineer $\rightarrow$ is equivalent to sub-senior. Metadata description of the Parameter quantification mapping table is shown in table 3.

Table 3 T_ZJ_SXLHYS : Parameter quantification mapping table

\begin{tabular}{|c|c|c|c|c|c|}
\hline Property & Name & Data type and length & Null or not & Primary/external key & remark \\
\hline F_ Id & Serial number & Integer & $\mathrm{N}$ & $\mathrm{P}$ & \\
\hline F_PJZBBH & $\begin{array}{l}\text { Number of the } \\
\text { evaluation index }\end{array}$ & Char(10) & $\mathrm{N}$ & & Value: Z11、Z12... \\
\hline F_LHXH & $\begin{array}{l}\text { Serial number of } \\
\text { quantification }\end{array}$ & Integer & $\mathrm{N}$ & & Value: 1 、 $2 \ldots$ \\
\hline F_SXBM & Attribute coding & & & & Exp:“title” based on \\
\hline
\end{tabular}




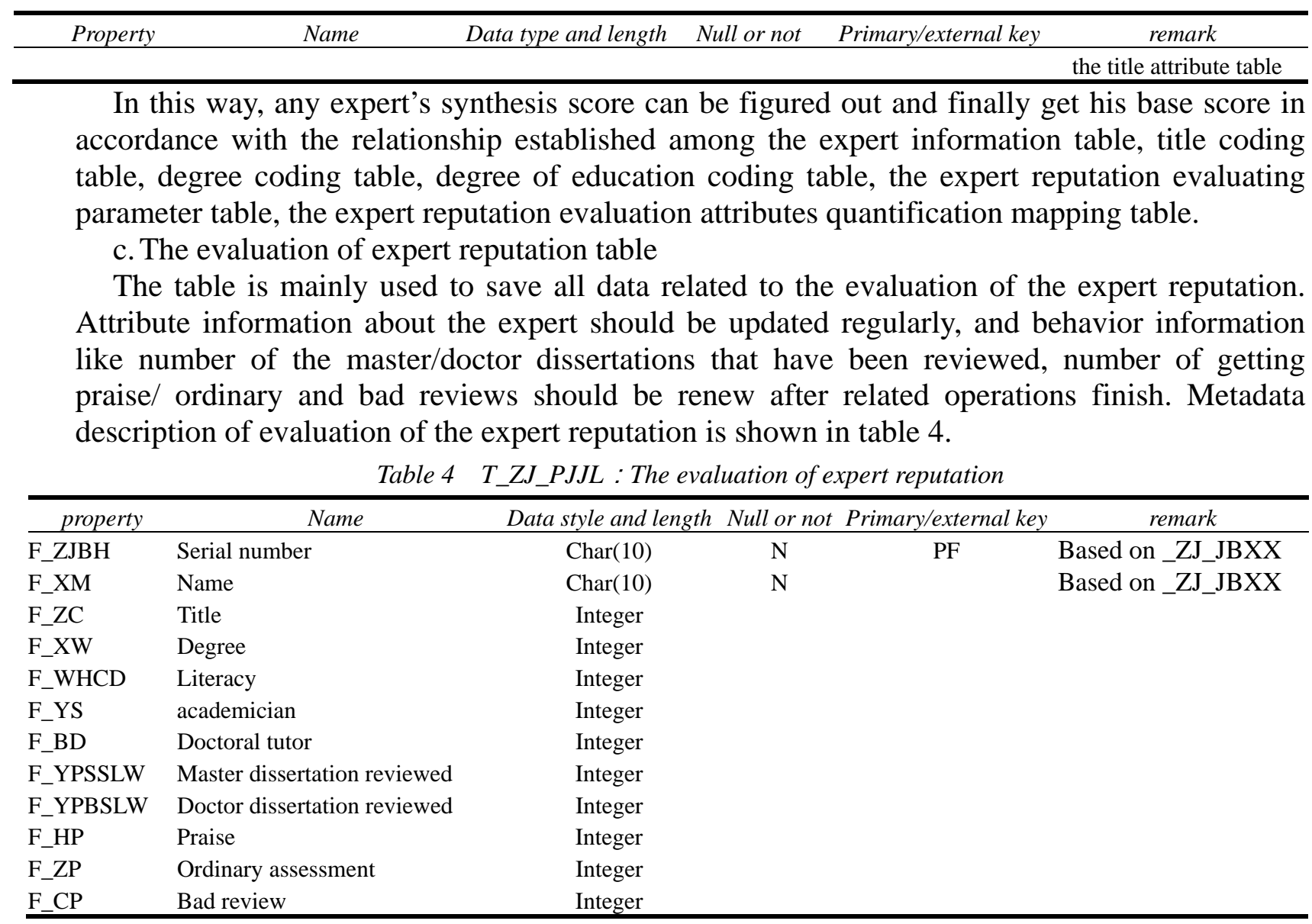

\section{Example of the evaluation of expert reputation}

1. Read the basic information about the expert:

Serial number:1010700689;Name:Wang xx; Title:professor; Degree:doctor;Degree of education: postgraduate; Academician: not; Doctoral tutor: not.

2. Get information about the evaluation experience

Master dissertations reviewed: 10; doctor dissertations reviewed: 2;

3. Get evaluation of the expert from organizers

Praise: 5; ordinary assessment: 6; Bad review: 1.

4. Calculate the base score

The initial score (S0) set by system to every expert is 50, so Wang XX's base score is:

$$
S 1=S 0+\sum_{i=1}^{5} S_{1 i}=50+10+10+5+0+0=75
$$

5. Calculate the experience score:

$$
S 2=S 21=S 21_{1} \times C 1+S 21_{2} \times C 2=1 \times 10+2 \times 2=14
$$

6. Calculate the evaluation score:

$$
\begin{aligned}
S 3=S 31 & =S 31_{1} \times P 1+S 31_{2} \times P 2+S 31_{3} \times P 3 \\
& =1 \times 5+0 \times 6+(-2) \times 1=3
\end{aligned}
$$

7. Calculate the synthesis score: $S=S 1+S 2+S 3=75+14+3=91$

\section{Summary}

It is clear that the evaluation of expert reputation system is simple in configuration, and has a little manual intervention. At the initial stage, expert reputation is based on the base score, after a period of time, the reputation is related to the experience score and the base score, and that also accords with the actual situation. By adjusting the initial parameter, the result of evaluation to the 
expert can be partly influenced, thus reflects the importance of the element of expert reputation. System saves basis data and history information about reputation and ability of the expert. Through the analysis of these data, we can have a clear sense about the expert.To some degree, the new evaluation system can make experts complete their evaluation more seriously, and the results can provide reliable reference for the management when selecting experts for evaluation.

\section{References}

[1] Rina Su. Practice and introspection about the anonymous system of the master's thesis evaluation[J]. Journal of inner Mongolia University of Technology, 2009,18(1):25-27 (in chinese).

[2] Yang J B,Xu D L.On the Evidential Reasoning Algorithm for Multiple Attribute Decision Analysis Under Uncertainty. IEEE Transactions on Systems Man and Cybernetics. 2002.

[3] Li Yi-Bo,Wang Ning,Zhou Chang.Based on D-S Evidence Theory of Information Fusion Improved Method. 2010.

[4] Yanfeng Ge, Guangxin Song. Research on online reputation evaluation under the electronic commerce erviroment[J]. Management, 2009(14) (in chinese).

[5] Yunan Zhan. Summary of relevant technology of recommending subsystem in online reputation management system[J]. Science \&Technology Information, 2009(15) (in chinese). 